\title{
The Use of Androgen Deprivation in Conjunction with Radiation in Localized Prostate Cancer
}

\author{
Omar Mahmoud* and May Abdel-Wahab*
}

Department of Radiation Oncology, Miller School of Medicine, University of Miami, 1475 NW 12th Ave (D-31), Miami, FL 33136, USA

\begin{abstract}
Background: In prostate carcinoma, there is controversy about optimal use of hormonal therapy with external beam radiotherapy: who will benefit, for how long, neo-adjuvant versus concurrent versus adjuvant.

Patients and Methods: Evidence from randomized studies in the light of recent published articles and updates were reviewed in order to address these issues.

Results and Conclusions: From this review it was concluded that the benefit of long-term hormonal therapy in combination with conventional-dose radiotherapy $(<74 \mathrm{~Gy})$ in high-risk prostate cancer is evident. For the intermediate risk, the evidence is still weak and studies are awaited to clarify this matter.
\end{abstract}

Keywords: External beam RT, prostate cancer, androgen suppression duration, androgen suppression timing.

\section{INTRODUCTION}

Prostate cancer $(\mathrm{PCa})$, with its lifetime risk of 1:6 men [1], constitutes an important health problem.

Many trials have shown an improved outcome when androgen suppression therapy (AST) is added to radiotherapy (RT) [2-6]. The general trend is to administer 6 months of AST for intermediate-risk patients and 2 to 3 years of treatment for patients with high-risk disease. However, increased recognition of specific side effects related to AST [7, 8] led to new questions regarding the indications for AST and the length of treatment needed.

\section{THE HISTORY AND MECHANISM OF ACTION OF HORMONE THERAPY IN PROSTATE}

In his Nobel Lecture 1966, Charles Huggins [9] showed that the hormone-dependent prostate cancer ( $\mathrm{PCa}$ ) cells undergo apoptosis if they are deprived of androgenic stimulation. As a consequence, depletion of circulating testosterone results in shrinkage of the prostate tumors and since then, AST has been used in the treatment of PCa patients.

In androgen-responsive murine adenocarcinoma from a Shinogi SC-115 cell lines, 89 Gy is required for $50 \%$ tumor control, however only $60 \mathrm{~Gy}$ is required to produce the same result if RT is combined with orchiectomy 1 day before RT. Also when RT preceded AST, the gain was no longer observed, pointing to the potential importance of timing and the advantage of neoadjuvant administration [10].

*Address correspondence to these authors at the Department of Radiation Oncology, Miller School of Medicine, University of Miami, 1475 NW 12th Ave (D-31), Miami, FL 33136, USA; Tel: 1-305-243-4337;

E-mail: mwahab@med.miami.edu and Department of Radiation Oncology, Tel: 1-727-259-1708; E-mail: omahmoud@med.miami.edu
AST promotes apoptosis and synergistically acts with radiation-induced killing of prostate cancer cells [11]. It also leads to reduction in the size of the prostate with the majority of reduction occurring within the first 3 months [12].

With this rational, clinical studies followed to answer the question of real benefit of combined modality as well as the dose and field issue.

\section{THE COMBINATION OF ADT \& RADIOTHERAPY IS BETTER THAN RADIOTHERAPY ALONE}

Several Phase III studies have demonstrated that combination of AST and radiotherapy is better than radiotherapy alone [2-6, 13-21].

It is important to note that combining RT and AST was found to be better than AST alone. Widmark et al. [22] reported on 875 locally advanced or high risk patients were randomized to total blockade for 3 months followed by Flutamide continuously versus treating with RT in combination with the same AST regimen. The cumulative incidence of PCa specific mortality at 10 years is $23.9 \%$ in endocrine therapy alone versus $11.9 \%$ in the combined modality arm, highlighting the importance of local therapy.

\section{EVIDENCE TO SUPPORT AST IN HIGH RISK PCA PATIENTS (TABLE 1)}

A recent update of a Swedish trial [19] randomizing 91 patients with locally advanced disease to RT (50 Gy to pelvis and 65 Gy to prostate) with or without orchiectomy was recently published. After up to 19 years follow-up, prostate cancer mortality ( $57 \%$ vs. $36 \%, p=0.02$ ) was significantly higher for the RT only arm. Subset analysis found that sur- 
Table 1. Trials Comparing Radiotherapy Alone Versus Combined Radiotherapy \& Androgen Suppression

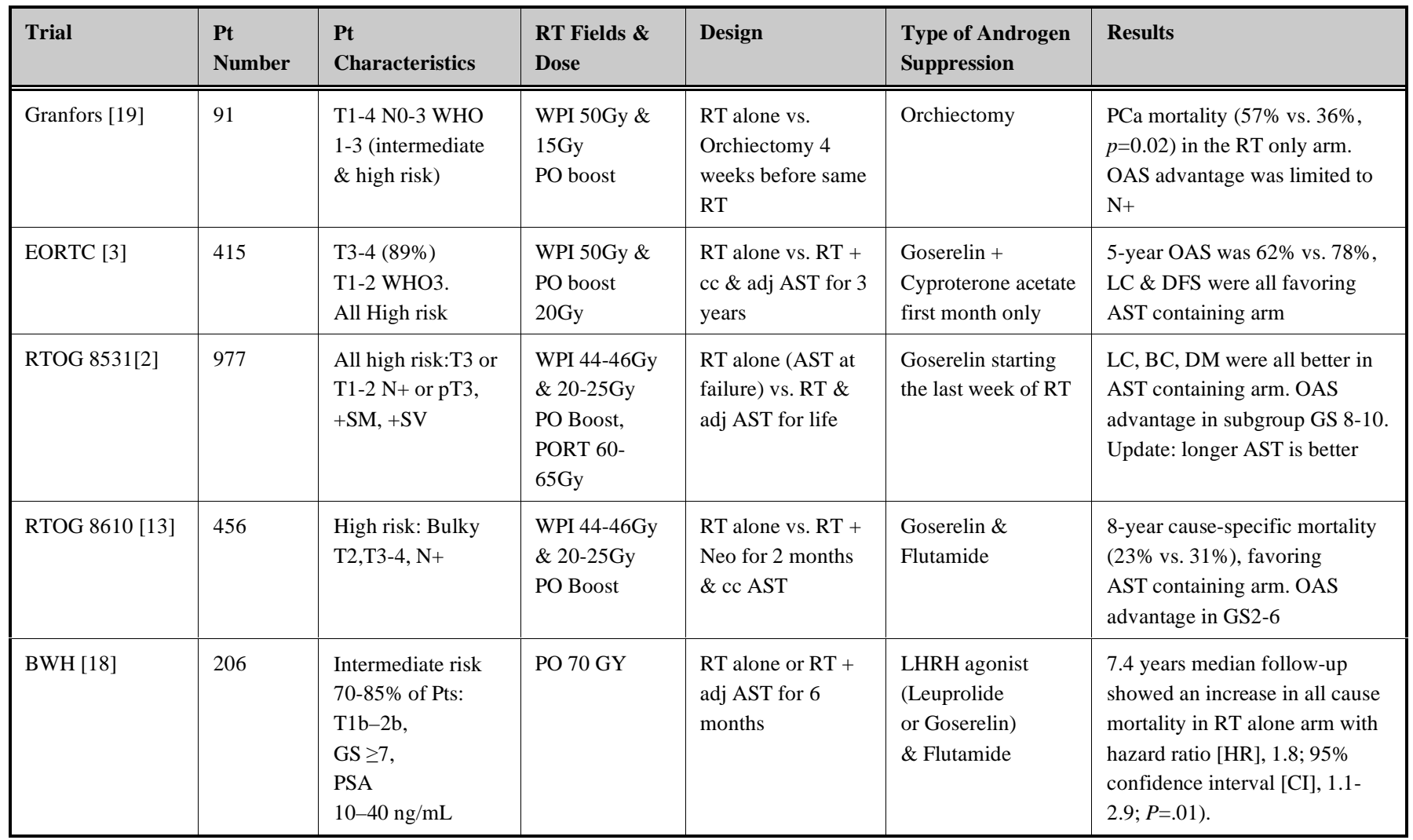

PCA=Prostate cancer, Pts - patients, EORTC - European Organization for Research and Treatment of Cancer, RTOG - Radiation Therapy Oncology Group, WHO -World Health Organization, BWH - Brigham and Women's Hospital (Boston, USA), BC - biochemical control, LC-local control, DFS-disease free survival, CSS-cancer specific survival, PFSprogression free survival, OAS-overall survival, DM - distant metastasis, SV - seminal vesicles, SM=Surgical Margin, RT - radiotherapy, WPI= Whole pelvis irradiation, $\mathrm{PO}=$ Prostate PORT - postoperative, PSA= Prostate specific antigen, GS - Gleason score Cc=Concurrent, Neo= Neoadjuvant, Adj=Adjuvant, $\mathrm{N}+=\mathrm{Lymph} \mathrm{Node}$ positive, LHRH= Luteinizing Hormone Releasing Hormone), AST= androgen suppression therapy.

vival advantage was limited to node positive patients detected by lymphadenectomy.

The EORTC 22863 trial [3] randomized high risk patients (T1-2 WHO grade 3 or T3-4 N0-1) to RT (50 Gy to pelvis and 20Gy to prostate and seminal vesicles) with or without immediate AST and for 3 years (Goserelin + Cyproterone acetate the first month only). With median follow-up of 66 months, 5-year overall survival was $62 \%(52-72)$ and $78 \%$ (72-84). In a recent update [23], the author found that the risk of cardiac death was $6 \%$ in combined treatment arm versus $4.2 \%$ in $\mathrm{RT}$ alone arm.

In RTOG 8531, 977 PCa patients with clinical or pathological stage T3 and/or nodal involvement either by radiographic evidence, or after radical prostatectomy, were randomized to either RT alone (with AST at relapse) or RT with AST (started in the last week of radiation and continued indefinitely). RT was 45 Gy to pelvis and 20-25Gy boost to prostate except in post-prostatectomy patients which formed $15 \%$ of patient population. Almost a third of the patients were Gleason 8-10 and almost a third had positive lymph nodes [2].

At 5.6 years median follow-up, 8-year local failure $23 \%$ vs. $37 \%(p<0.0001)$, distant metastases $27 \%$ vs. $37 \%$ $(p<0.0001)$, and PSA control $32 \%$ vs. $8 \%(p<0.0001)$ were in favor of the combined treatment arm [2]. Overall, there was no difference in overall survival or cause-specific survival. However, in subgroup analyses [24], an improvement in overall survival $(p=0.036)$ and cause-specific mortality $(p=0.019)$ was observed in the high risk group (Gleason 8-10).

An update [25] divided patients into three group based on the length of AST (Goserelin) into three groups: less than 1 year, 1 to 5 years, more than 5 years. At median follow-up of 9.6 years and of the 189 analyzable surviving patients, overall survival, disease free survival and fewer distant metastasis were better in the group of more than 5 years AST. Even after adjusting for other variables, AST more than 5 years remained significant for the studied end points. Another report pointing that long-term AST improve survival.

The RTOG 8610 study [13] included 456 men, T3-4 formed $70 \%$ and Gleason 8-10 formed $28 \%$ of the patient population. They were randomized to RT alone versus AST in addition to RT. AST consisted of 2 months of neoadjuvant AST in addition to AST concurrent with RT or. RT was 4446 Gy to pelvis, followed by $20-25$ Gy boost to prostate. After a median follow-up of 6.7 years, 8-year cause-specific mortality $(23 \%$ vs. $31 \%, p=0.05)$ favoring combined treatment arm. Paradoxically and contrary to RTOG 8531, all endpoints including overall survival ( $70 \%$ vs. $52 \%, p=0.015)$ were improved in Gleason 2-6. 


\section{RADIOTHERAPY DOSE \& FIELDS WITH AST}

In spite of the evidence favoring the use of AST with RT, it is important to note that most dose escalation studies did not incorporate AST [26-32]. Lab studies hinted toward effectiveness of lower dose of RT with neoadjuvant AST [10]. Thus it remains to be seen if AST can make up for a lower RT dose or make up for a smaller field?

One of the very few studies to combine AST with dose escalation, the MRC RT01 trial [33] compared 64 and 74 Gy to the prostate after 3-6 months of neoadjuvant AST. 843 PCa patients with a median PSA $12.8 \mathrm{ng} / \mathrm{ml}$, Gleason score $8-10$ in $13 \%$ \& $13 \%$ had T3 were followed for a median of 63 months. The 5 year biochemical control was 71 versus 61 $\%(\mathrm{p}=0.0007)$ in favor of higher dose.

Two trials addressed the question of whether whole pelvis radiotherapy was needed in patients whose estimated risk of pelvic lymph node involvement was higher than $15 \%$.

The first of these 2 trials: RTOG 9413 a $2 \times 2$ randomization study [15] enrolled 1,292 men. Eligible patients had a PSA level of $\leq 100 \mathrm{ng} / \mathrm{ml}$ and an estimated risk of lymph node involvement of $>15 \%$. Patients with cT2c-4 and Gleason score $\leq 6$, were eligible even if their calculated risk of lymph node involvement did not reach $15 \%$. They were randomized to either four months of neoadjuvant and concurrent (NCHT) or 4 months of adjuvant (AHT) androgen suppression therapy, and whole pelvic radiotherapy (WPRT) to 50.4 Gy and a boost 19.8 Gy to prostate or prostate-only radiotherapy (PORT) to $70.2 \mathrm{~Gy}$. All RT was given at $1.8 \mathrm{~Gy}$ per fraction. The 4-year progression-free survival was 54\% and $47 \%$ for the WPRT and PORT arms, respectively $(p=0.02)$. No difference in 4-year progression-free survival was seen between the NCHT and AHT arms $(p=0.56)$.

To analyze the effect of the RT field size and to avoid systemic treatment completion variable, a secondary analysis [34] of two of the four arms was done. Only the NCHT arms were studied, allowing the comparison of WPRT versus PORT. PORT arm was further divided into 2 subgroups the "mini-pelvis group" $(>10 \times 11 \mathrm{~cm})$, and the "prostate-only group" $(<10 \times 11 \mathrm{~cm})$. As expected and confirming initial publication, improved 4-year progression free survival was significantly associated with larger field size $(p=0.024)$ at the expense of higher acute grade 2 or greater GI toxicities as well as grade 3 late GI toxicities which were more frequent with larger fields (WPRT, "mini-pelvis", prostateonly: $46.6 \%, 36.7 \%, 20.2 \%, p<0.001)$. It is important to note that the study used conventional RT techniques rather than IMRT (intensity modulated radiation therapy) which may have adversely affected the toxicity profile.

In a recent update [35] of the RTOG 9413, a median follow-up of 79 months was reached. Interestingly, the initial improvement in progression-free survival seen in the WPRT versus the PORT was lost with longer follow-up ( $p=0.99)$. Also, no difference was seen between the NCHT and AHT arms $(p=0.59)$ [35]. On a closer look, WPRT and AHT had a trend for being the least effective arm. When comparing WPRT and AHT versus WPRT and NHT; NHT is significantly better for progression free survival $(\mathrm{P}=0.014)$. Also in NHT when comparing WPRT vs. PORT, again WPRT had a trend towards better results with $\mathrm{p}=0.023$. This Pair wise comparison must be viewed with caution as $2 \times 2$ factorial designs preclude analysis of significant interaction between arms.

The second trial GETUG-01, a French trial [36], randomized $444 \mathrm{~T} 1 \mathrm{~b}-3 \mathrm{~N} 0$ patients, with any PSA, and any Gleason score to WPRT or PORT. Neoadjuvant and concurrent AST for 4-8 months was used in high-risk patients (T3, Gleason score $\geq 7$, or PSA $>12$ ). 66-70 Gy was delivered to the prostate. In the trial, almost half of the patients were low risk. No significant difference was seen in 5 year progression free survival between both arms, even for high risk patients.

It must be noted that all AST trials used suboptimal doses in the RT alone arm which was in the range of 66-70Gy. It is not clear whether dose escalation alone would be sufficient to attain equal results as AST and RT.

\section{OPTIMAL TIMING AND DURATION OF AST (TABLE 2)}

Lab data [10] and the initial analysis of RTOG 9413 suggest that neoadjuvant AST might be better [15], although this observation did not hold on subsequent analysis [35] or in some other studies [16-17].

In RTOG 9413, which included high risk patients, the duration of either arms NCHT or AHT (4months) was suboptimal to view a benefit as suggested from other trials [14-22, 26-45] that used long-term AST in this patient population. So, a design that incorporates long-term adjuvant AST, with or without neoadjuvant is needed in the high risk category to better elucidate this issue.

Two successive studies from Quebec were conducted to shed light on the issue of timing and duration of AST. The first L-101 randomized $120 \mathrm{~T} 2-3 \mathrm{PCa}$ patients, to RT alone 64 Gy (prostate only) versus RT and neoadjuvant AST (LHRH agonist \& Flutamide) for 3 months, versus RT with neoadjuvant, concurrent and adjuvant AST for 10 months [16]. Assessment was by TRUS guided biopsy at 12 and 24 months after the completion of RT. At 12 months, 62\%, $30 \%$ and $4 \%$ of patients had positive biopsies in the RT, RT and neoadjuvant ( 3 months AST) and RT with 10 months AST groups respectively. Also at 24 months, 65\%, 28\% and $5 \%$ had biopsies that were positive for residual disease in the respective groups above. PSA measurement correlated with biopsy results at 12 months but did not differ between group 2 and 3 at 24 months. These results suggest that AST and RT may be better than RT alone in high risk patients, who constituted about third of the study population. However, the timing of AST needs further clarification as well as the duration in each patient risk category.

That's why L-200 trial was carried out. The study enrolled 325 patients as opposed to the smaller number (120 patients) enrolled in the L-101. The L-200 study attempted to answer the question of whether 5 versus 10 months of neoadjuvant, concurrent \& adjuvant AST with LHRH +Flutamide made a difference in biochemical no evidence of disease (BNED) outcomes. In the L-200 trial, BNED at 4 years was $65 \%$ with no difference between 5 and 10 months of AST. Thus, these 2 studies confirm, the lack of a difference between 5 and 10 months of AST and between different timing of AST in this patient population [17]. 
Table 2. Trials Comparing Different Timing \& Duration of Androgen Suppression Therapy with Radiotherapy

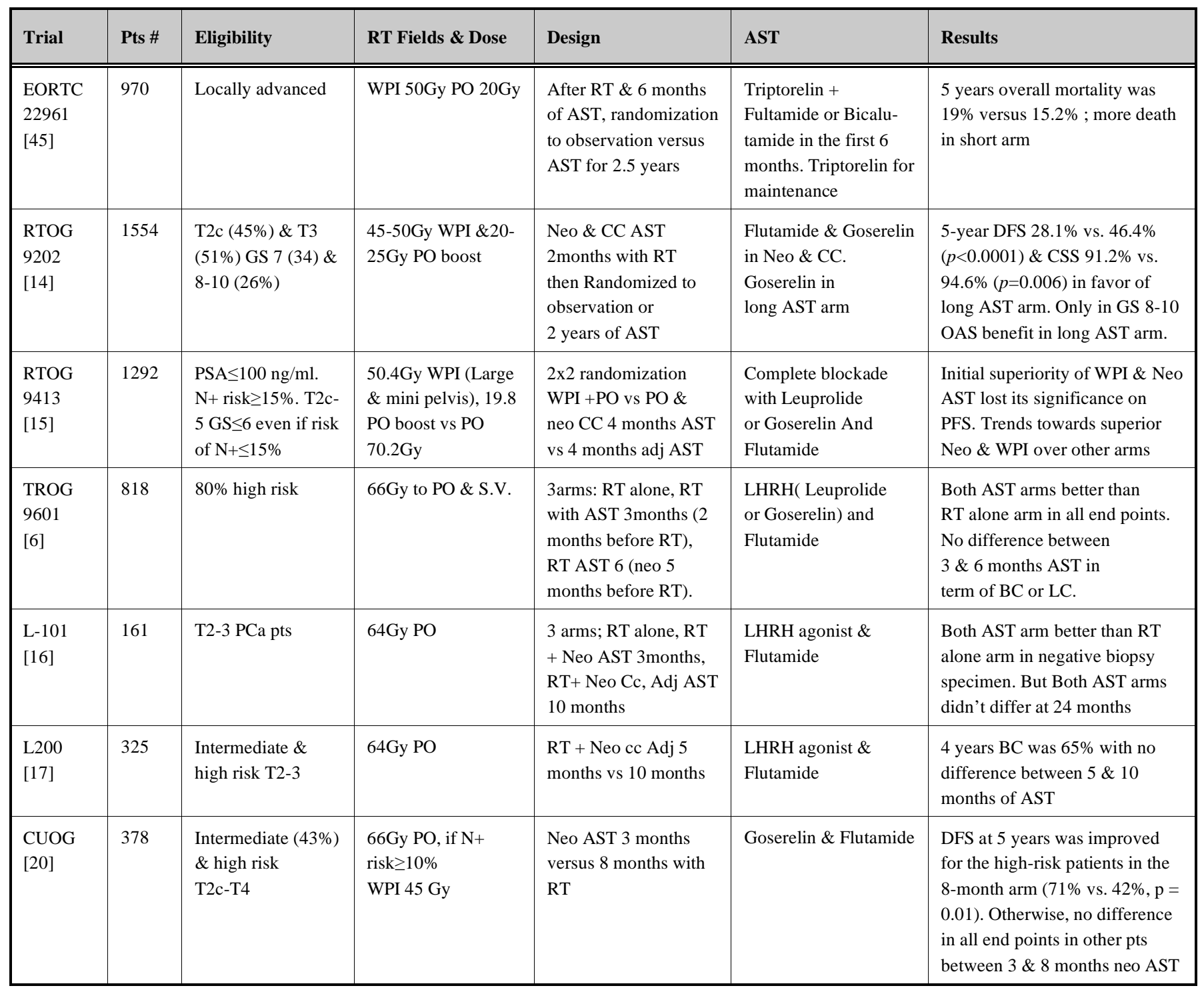

PCA=Prostate cancer, Pts - patients, EORTC - European Organization for Research and Treatment of Cancer, RTOG - Radiation Therapy Oncology Group, WHO -World Health Organization, BWH - Brigham and Women's Hospital (Boston, USA), BC - biochemical control, LC-local control, DFS-disease free survival, CSS-cancer specific survival, PFSprogression free survival, OAS-overall survival, DM - distant metastasis, SV - seminal vesicles, SM=Surgical Margin, RT - radiotherapy, WPI= Whole pelvis irradiation, $\mathrm{PO}=$ Prostate PORT - postoperative, PSA= Prostate specific antigen, GS - Gleason score. $\mathrm{Cc}=$ Concurrent, Neo= Neoadjuvant, Adj=Adjuvant, N+=Lymph Node positive, LHRH= Luteinizing Hormone Releasing Hormone), AST= androgen suppression therapy

One of the possible explanations was that the percentage of high risk patients, who are known to benefit from longer AST, was small. This explanation is further supported by data showing, the lack of additional benefit to longer AST in the intermediate risk population. Such as that from the Canadian study [20] which confirmed that longer versus shorter neoadjuvant AST is no benefit in intermediate risk population.

378 PCa men with T1c-4, any PSA, and any Gleason score were randomized in the Canadian trial [20] to either 3 or 8 months of neoadjuvant AST (Goserelin and Flutamide) prior to radiotherapy (66-67 Gy to the prostate). Patients with an estimated nodal involvement of $>10-15 \%$ received whole-pelvic radiotherapy (45-46 Gy). Low risk population constituted $26 \%$ while the intermediate risk group constituted $43 \%$ of the study population. Median follow-up was 44 months. No significant difference was noted in any of the end points [20]. An update published in 2009 with 6.6 years median follow-up [37], again showed no difference in all end points with the exception of high risk patients that benefited from longer AST with an improved disease-free survival rate at 5 years compared to the 8 -month arm $(71 \%$ vs. $42 \%, \mathrm{p}=$ $0.01)$.

\section{OPTIMAL DURATION OF NEOADJUVANT AST}

From Australia [6], TROG 9601 randomized $818 \mathrm{PCa}$ patients to one of three arms: RT alone, RT with 3 months AST (LHRH agonist + Flutamide) (started 2 months before radiotherapy), or RT with 6 months of AST (started 5 months before radiotherapy). Radiotherapy was delivered the prostate and seminal vesicles to a total dose of $66 \mathrm{~Gy}$ in 33 fractions. More than $80 \%$ of patients qualified as high risk. 
At a median follow-up of 5.9 years, 3 months of AST significantly improved biochemical control (HR 0.70, $p=0.002)$, and disease-free survival (HR $0.65, p=0.0001$ ) when compared to RT alone. However, a longer AST duration of 6 months not only significantly improved biochemical control (HR 0.58, $p<0.0001$ ) and disease-free survival (HR 0.56, $p<0.0001$ ), but also distant failure (HR 0.67, $p=0.046$ ), and prostate cancer specific survival (HR 0.56, $p=0.04$ ) when compared to RT. On the other hand, no difference in local control or biochemical free survival was noticed between the 3 and 6 month groups. Subgroup analysis for the benefit of AST in intermediate risk showed no significant DFS with $\mathrm{HR}=0.8$ in favor of AST but the CI was wide (0.45-1.42).

The Canadian [20] and Australian [6] trials are inconsistent in patient population and in results, however, they suggest that longer AST is beneficial in high risk patients and of minimal benefit for intermediate risk patients.

\section{AST IN INTERMEDIATE RISK PATIENTS}

Data regarding AST are limited in the intermediate risk population. Furthermore, the optimum duration, and need for AST are controversial in the setting of dose escalation.

D'Amico trial [18] randomized 206 patients with mostly (70-85\%) intermediate risk prostate cancer to radiotherapy alone or radiotherapy with 6 months of androgen suppression therapy (AST) (LHRH agonist and Flutamide, both for 6 months). The study population had a median PSA of $11 \mathrm{ng} /$ $\mathrm{ml}$, a PSA velocity more than 2ng/ml/year; clinical T1 $(48 \%)$ and T2 $(52 \%)$ disease and a range of Gleason scores from $<7$ (27\%) and $7(58 \%)$, to 8-10 (15\%). Conformal radiotherapy was used to treat the prostate only delivering $70 \mathrm{~Gy}$ over 35 fractions. At a median follow-up of 4.52 years, the combined arm did better with 5-year overall survival (88\% vs. $78 \%$, $p=0.04)$, cause-specific survival (100\% vs. 94\%, $p=0.02)$, and PSA control (79\% vs. 55\%, $p<0.001)$. The update [5] of this trial after 7.4 years median follow-up showed an increase in all cause mortality in radiotherapy alone arm with hazard ratio $[\mathrm{HR}], 1.8 ; 95 \%$ confidence interval $[\mathrm{CI}], 1.1$ $2.9 ; P=.01)$. Assessment of co-morbidities and their interaction with the results was added to this update: In the 157 men with no or minimal co morbidity scores, treatment with RT and AST compared with RT was associated with a significantly higher survival; HR, 4.2; 95\% CI, 2.1-8.5; $\left.P_{-} .001\right)$, with Kaplan-Meier 8-year survival estimates of $90 \%(95 \%$ CI, 79\%-95\%) and 64\% (95\% CI, 49\%-75\%), respectively.

The take home message from the update is that, men with little co-morbidity, intermediate risk PCa and PSA velocity more than $2 \mathrm{ng} / \mathrm{ml} / \mathrm{year}$ benefit from abbreviated course of androgen suppression.

Results of the RTOG 9910 and RTOG 9406 studies, which included a majority of patients in the low or intermediate risk group, are awaited to shed light on the need for and the duration of AST in this particular population.

\section{LONGER VERSUS SHORTER ADJUVANT AST IN HIGH RISK PATIENTS}

The prolonged use of AST, especially in older patients, may lead to several complications, including decline in bone density, osteoporosis, fractures [38] decrease in muscle strength [39, 40] mental changes [41] and metabolic syn- drome and cardiac toxicity [42] with a significant impact in patients' quality of life [43].

This concern led to a combined analysis of the 3 studies led by D'Amico [18], Denham [6] and Bolla [3] to compare short versus long-term AST. After adjusting for known prognostic factors in high risk patients, long-term AST did not result in significant prolongation of survival in older men [44].

More interestingly, the long-awaited EORTC 22961 was recently published [45] to answer the question of long versus short-term AST. In this context, 970 locally advanced PCa patients were randomized after Conformal RT (50 Gy to pelvis \& $20 \mathrm{~Gy}$ to prostate and seminal vesicles) and 6 months AST (LHRH agonist Triptorelin and anti-androgen Flutamide or Bicalutamide) to observation versus continued AST with Triptorelin for 2.5 years. After median follow-up of 6.6 years, the 5 years overall mortality was $19 \%$ versus $15.2 \%$ and hazard ratio was 1.42 in short versus long-term AST with $\mathrm{p}=0.6$ for non inferiority of the short arm, which confirms that shorter AST provides inferior overall survival results. Importantly, no difference in death from other causes was noted between the 2 arms, including fatal cardiac myocardial infarction.

1,554 men with high risk PCa, T2c (45\%) \& T3 (51\%), Gleason score 7 in $34 \%$ \& 8-10 in $26 \%$ were randomized in RTOG 9202 after 2 months of neoadjuvant and concurrent hormones with radiotherapy to either observation or 2 years of adjuvant hormones [14]. RT was 45-50 Gy to pelvis and 20-25 Gy to prostate boost. At 5.8 years median follow-up, the 5-year disease-free survival was $28.1 \%$ vs. $46.4 \%$ $(p<0.0001)$ and cause-specific survival was $91.2 \%$ vs. $94.6 \%$ $(p=0.006)$, in favor of the 2 year adjuvant hormone arm. Overall survival, was significantly improved in the adjuvant hormones arm, but only in Gleason score 8-10 subset, The update after 10 years [46] showed again that in the high risk group with Gleason score 8-10, long-term adjuvant AST improve survival $31.9 \%$ versus $45.1 \%(P=0.0061)$.

\section{CONCLUSION AND FUTURE PERSPECTIVES}

AST has an important role in the treatment of high risk prostate cancer patients in the setting of definitive radiation therapy with conventional doses. Some benefits can also be seen in select intermediate risk patients. Less information is available regarding the use of AST in the dose-escalation setting.

The future brings new perspectives and new exciting discoveries. Identification of high risk patients based on the molecular background rather that actual risk stratification will further refine treatment decisions regarding who will benefit from AST and how to prevent relapse [47]. Advanced imaging techniques to help detect early lymph node spread may have a role in future recommendations for AST. New markers will better segregate completely eradicated PCa from those who still have disease and who will benefit more from other form of treatment.

Chemotherapy and new targeted therapies may eventually add to the management of high risk patients.

Tumor vaccine as well as new anti androgen and other may prove to be more efficient. 


\section{REFERENCES}

[1] Jemal A, Siegel R, Ward E, et al. Cancer statistics. CA Cancer J Clin 2006; 56: 106-30.

[2] Pilepich M, Winter K, Lawton C, et al. Androgen suppression adjuvant to definitive radiotherapy in prostate carcinoma- longterm results of phase III RTOG 85-31. Int J Radiat Oncol Biol Phys 2005; 61: 1285-90.

[3] Bolla M, Collette L, Blank L, et al. Long-term results with immediate androgen suppression and external irradiation in patients with locally advanced prostate cancer (an EORTC study): a phase III randomized trial. Lancet 2002; 360: 103-6.

[4] Horwitz E, Bae K, Hanks G, et al. Ten-year follow-up of radiation therapy oncology group protocol 92-02: a phase III trial of the duration of elective androgen deprivation in locally advanced prostate cancer. J Clin Oncol 2008; 26: 2497-504.

[5] D'Amico A, Chen M, Renshaw A, et al. Androgen suppression and radiation $v s$. radiation alone for prostate cancer: a randomized trial. JAMA 2008; 299: 289-95.

[6] Denham J, Steigler A, Lamb D, et al. Short-term androgen deprivation and radiotherapy for locally advanced prostate cancer: results from the Trans-Tasman Radiation Oncology Group 96.01 randomized controlled trial. Lancet Oncol 2005; 6: 841-50.

[7] Keating N, O'Mally A, Smith M. Diabetes and cardiovascular disease during androgen deprivation therapy for prostate cancer. $\mathbf{J}$ Clin Oncol 2006; 24: 4448-56.

[8] D'Amico A, Denham J, Crook J, et al. Influence of androgen suppression therapy for prostate cancer on the frequency and timing of fatal myocardial infractions. J Clin Oncol 2007; 25: 2420-5.

[9] Huggins C. Endocrine-induced regression of cancers. Nobel Lecture [Available on] http://nobelprize.org/nobel prizes/medicine/ laureates/1996/huggins-lecture.html

[10] Zietman AL, Nakfoor BM, Prince EA, et al. The effect of androgen deprivation and radiation therapy on an androgen sensitive murine tumor: an in vitro and in vivo study. Cancer J Sci Am 1997; 3: 31.

[11] Joon DL, Hasegawa M, Sikes C, et al. Supra-additive apoptotic response of R3327-G rat prostate tumors to androgen ablation and radiation. Int J Radiat Oncol Biol Phys 1997; 38: 1071-7.

[12] Lilleby W, Fossa SD, Knutsen BH, et al. Computed tomography/magnetic resonance based volume changes of the primary tumour in patients with prostate cancer with or without androgen deprivation. Radiother Oncol 2000; 57: 195-200.

[13] Pilepich MV, Winter K, John MJ, et al. Phase III Radiation Therapy Oncology Group (RTOG) trial 86-10 of androgen deprivation adjuvant to definitive radiotherapy in locally advanced carcinoma of the prostate. Int J Radiat Oncol Biol Phys 2001; 50: 1243-52.

[14] Hanks GE, Pajak TF, Porter A, et al. Phase III trial of long-term adjuvant androgen deprivation after neoadjuvant hormonal cytoreduction and radiotherapy in locally advanced carcinoma of the prostate: The Radiation Therapy Oncology Group Protocol 92-02. J Clin Oncol 2003; 21: 3972-8.

[15] Roach M III M, DeSilvio M, Lawton C, et al. A phase III trial comparing whole pelvic versus prostate only radiotherapy and neoadjuvant concurrent versus adjuvant combined androgen suppression: Radiation Therapy Oncology Group (RTOG) 9413. J Clin Oncol 2003; 21: 1904-11.

[16] Lavardiere J, Gomez JL, Cusan L, et al. Beneficial effect of combination hormonal therapy administered prior and following external beam radiation therapy in localized prostate cancer. Int J Radiat Oncol Biol Phys 1997; 7: 247-52.

[17] Laverdiere J, Nabid A, De Bedoya LD, et al. The efficacy and sequencing of a short course of androgen suppression on freedom from biochemical failure when administered with radiation therapy for T2-T3 prostate cancer. J Urol 2004; 171: 1137-40.

[18] D'Amico AV, Manola J, Loffredo M, et al. 6-month androgen suppression plus radiation therapy vs. radiation therapy alone for patients with clinically localized prostate cancer: a randomized controlled trial. JAMA 2004; 292: 821-7.

[19] Granfors RiT, Modig H, Damber JE, Tomic R. Long-term followup of a randomized study of locally advanced prostate cancer treated with combined orchiectomy and external radiotherapy versus radiotherapy alone. J Urol 2006; 176: 544-7.

[20] Crook J, Ludgate C, Malone S, et al. Report of a multicenter Canadian phase III trial of 3 months vs 8 months neoadjuvant androgen before standard dose radiotherapy for clinically localized prostate cancer. Int J Radiat Oncol Biol Phys 2004; 60: 15-23.
[21] Denham JW, Steigler A, Lamb DS, et al. Short-term androgen deprivation and radiotherapy for locally advanced prostate cancer: results from the Trans-Tasman Radiation Oncology Group 96.01 randomized controlled trial. Lancet Oncol 2005; 11: 84150 .

[22] Widmark A, Klepp O, Solberg A, et al. Scandinavian Prostate Cancer Group Study 7; Swedish Association for Urological Oncology 3. Endocrine treatment, with or without radiotherapy, in locally advanced prostate cancer (SPCG-7/SFUO-3): an open randomised phase III trial. Lancet 2009; 373(9660): 301-8.

[23] Bolla M, Collette L, Van Tienhoven G, et al. Ten-year update of long-term adjuvant androgen deprivation with gosereline in patients with locally advanced prostate cancer treated with radiotherapy: a phase III EORTC study. Int J Radiat Oncol Biol Phys 2008; 72(Suppl 1): S30-1.

[24] Lawton CA, Winter K, Murray K, et al. Updated results of the phase III Radiation Therapy Oncology Group (RTOG) trial 85-31 evaluating the potential benefit of androgen suppression following standard radiation therapy for unfavorable prognosis carcinoma of the prostate. Int J Radiat Oncol Biol Phys 2001; 49(4): 937-46.

[25] Souhami L, Bae W, Pilepich M, and Sandler H. Impact of the duration of adjuvant hormonal therapy in patients with locally advanced prostate cancer treated with radiotherapy: a secondary analysis of RTOG 85-31. J Clin Oncol 2009; 27: 2137-43.

[26] Dearnaley DP, Hall E, Lawrence D, et al. Phase III pilot study of dose escalation using conformal radiotherapy in prostate cancer: PSA control and side effects. Br J Cancer 2005; 92: 488-98.

[27] Lukka H, Hayter C, Julian JA, et al. Randomized trial comparing two fractionation schedules for patients with localized prostate cancer. J Clin Oncol 2005; 23: 6132-8.

[28] Peeters STH, Heemsbergen WD, Koper PCM, et al. Dose response in radiotherapy for localized prostate cancer: Results of the Dutch multicenter randomized phase III trial comparing 68 Gy of radiotherapy with 78 Gy. J Clin Oncol 2006; 24: 1990-6.

[29] Sathya JR, Davis IR, Julian JA, et al. Randomized trial comparing iridium implant plus external-beam radiation therapy with externalbeam radiation therapy alone in node-negative locally advanced cancer of the prostate. J Clin Oncol 2005; 23: 1192-9.

[30] Shipley WU, Verhey LJ, Munzenrider JE, et al. Advanced prostate cancer: the results of a randomized comparative trial of high dose irradiation boosting with conformal protons compared with conventional dose irradiation using photons alone. Int J Radiat Oncol Biol Phys 1995; 32: 3-12.

[31] Zietman AL, DeSilvio ML, Slater JD, et al. Comparison of conventional-dose vs high-dose conformal radiation therapy in clinically localized adenocarcinoma of the prostate: a randomized controlled trial. JAMA 2005; 294: 1233-9.

[32] Pollack A, Zagars GK, Starkschall G, et al. Prostate cancer radiation dose response: Results of the $\mathrm{M}$. D. Anderson phase III randomized trial. Int J Radiat Oncol Biol Phys 2002; 53: 1097 105.

[33] Dearnaley DP, Sydes MR, Graham JD, et al. Escalated-dose versus standard-dose conformal radiotherapy in prostate cancer: first results from the MRC RT01 randomised controlled trial. Lancet Oncol 2007; 8(6): 475-87.

[34] Roach M 3rd, DeSilvio M, Valicenti R, et al. Whole-pelvis, "minipelvis," or prostate-only external beam radiotherapy after neoadjuvant and concurrent hormonal therapy in patients treated in the Radiation Therapy Oncology Group 9413 trial. Int J Radiat Oncol Biol Phys 2006; 66(3): 647-53.

[35] Lawton CA, DeSilvio M, Roach M $3^{\text {rd }}$, et al. An update of the phase III trial comparing whole pelvic to prostate only radiotherapy and neoadjuvant to adjuvant total androgen suppression: updated analysis of RTOG 94-13, with emphasis on unexpected hormone/radiation interactions. Int J Radiat Oncol Biol Phys 2007; 69(3): 646-55.

[36] Pommier P, Chabaud S, Lagrange JL, et al. Is there a role for pelvic irradiation in localized prostate adenocarcinoma? Preliminary results of GETUG-01. J Clin Oncol 2007; 25(34): 5366-73.

[37] Crook J, Ludgate C, Malone S, et al. Final report of multicenter Canadian Phase III randomized trial of 3 versus 8 months of neoadjuvant androgen deprivation therapy before conventional-dose radiotherapy for clinically localized prostate cancer. Int J Radiat Oncol Biol Phys 2009; 73(2): 327-33. 
[38] Daniell HW, Dunn SR, Ferguson DW, et al. Progressive osteoporosis during androgen deprivation therapy for prostate cancer. $\mathbf{J}$ Urol 2000; 163: 181-6.

[39] Higano CS. Side effects of androgen deprivation therapy: Monitoring and minimizing toxicity. Urology 2003; 61:32-8.

[40] Smith MR, Finkelstein JS, McGovern FJ, et al. Changes in body composition during androgen deprivation therapy for prostate cancer. J Clin Endocrinol Metab 2003; 87: 599-603.

[41] Jenkins VA, Bloomfield DJ, Shilling VM, et al. Does neoadjuvant hormone therapy for early prostate cancer affect cognition? Results from a pilot study. BJU Int 2005; 96: 48-53.

[42] Tsai HK, D’Amico AV, Sadetsky N, et al. Androgen deprivation therapy for localized prostate cancer and the risk of cardiovascular mortality. J Natl Cancer Inst 2007; 99: 1516-24.

[43] Braga-Basaria M, Dobs AS, Muller DC, et al. Metabolic syndrome in men with prostate cancer undergoing long-term androgendeprivation therapy. J Clin Oncol 2006; 24: 3979-83.
[44] D'Amico AV, Denham JW, Bolla M, Collette L, Lamb DS, Tai $\mathrm{KH}$, Steigler A, Chen MH. Short- vs long-term androgen suppression plus external beam radiation therapy and survival in men of advanced age with node-negative high-risk adenocarcinoma of the prostate. Cancer 2007; 109(10): 2004-10.

[45] Bolla M, de Reijke TM, Van Tienhoven G, et al. Duration of androgen suppression in the treatment of prostate cancer. N Engl $\mathrm{J}$ Med 2009; 360(24): 2516-27.

[46] Horwitz EM, Bae K, Hanks GE, et al. Ten-year follow-up of radiation therapy oncology group protocol 92-02: a phase iii trial of the duration of elective androgen deprivation in locally advanced prostate cancer. J Clin Oncol 2008; 26: 2497-504.

[47] Abdel-Wahab M, Berkey B, Krishan A, et al. Influence of number of CAG repeats on local control in the RTOG 86-10 protocol. Am J Clin Oncol 2006; 29(1): 14-20.

(C) Mahmoud and Abdel-Wahab; Licensee Bentham Open

This is an open access article licensed under the terms of the Creative Commons Attribution Non-Commercial License (http://creativecommons.org/licenses/ by-nc/3.0/) which permits unrestricted, non-commercial use, distribution and reproduction in any medium, provided the work is properly cited. 